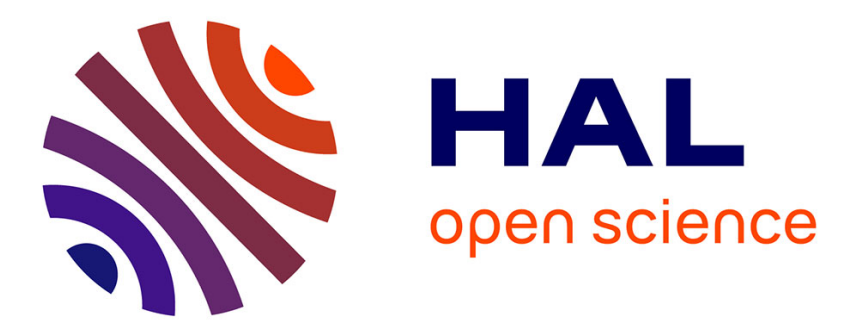

\title{
From discrete and iterative deconvolution operators to machine learning for premixed turbulent combustion modeling.
}

\author{
Pascale Domingo, Zacharias Nikolaou, Andréa Seltz, Luc Vervisch
}

\section{- To cite this version:}

Pascale Domingo, Zacharias Nikolaou, Andréa Seltz, Luc Vervisch. From discrete and iterative deconvolution operators to machine learning for premixed turbulent combustion modeling.. Data analysis for direct numerical simulation of turbulent combustion, 2020, 10.1007/978-3-030-44718-2_11 . hal03042541

\section{HAL Id: hal-03042541}

https://hal-normandie-univ.archives-ouvertes.fr/hal-03042541

Submitted on 6 Dec 2020

HAL is a multi-disciplinary open access archive for the deposit and dissemination of scientific research documents, whether they are published or not. The documents may come from teaching and research institutions in France or abroad, or from public or private research centers.
L'archive ouverte pluridisciplinaire HAL, est destinée au dépôt et à la diffusion de documents scientifiques de niveau recherche, publiés ou non, émanant des établissements d'enseignement et de recherche français ou étrangers, des laboratoires publics ou privés. 


\title{
From discrete and iterative deconvolution operators to machine learning for premixed turbulent combustion modeling
}

\author{
P. Domingo, Z. Nikolaou, A. Seltz, L. Vervisch
}

\begin{abstract}
Following the rapid and continuous progress of computing power allowing for increasing the mesh resolution in large eddy simulation (LES), new modeling strategies appear which are based on a direct treatment of the now well-resolved, but still not fully-resolved scalar signals. Along this line, deconvolution or inverse filtering, either based on discrete or iterative operators, is first discussed. Recent results obtained from a direct numerical simulation (DNS) database and LES of a premixed turbulent jet flame are presented. The analysis confirms the potential of deconvolution to approximate the unclosed non-linear terms and the SGS fluxes. Then, the introduction of machine learning in turbulent combustion modeling is illustrated in the context of convolutional neural networks.
\end{abstract}

\section{Introduction}

Virtual prototyping has become an essential ingredient in the development and optimisation of combustion systems, as furnaces, boilers, internal combustion engines and gas turbines $[1,2,3,4,5]$. Even relying on high performance computing, the spatial resolutions reached by the grids do not allow for fully resolving the smallest scales of the flow motions and scalar signals when simulating real combustion chambers. The introduction of modeling is therefore mandatory to deal with unresolved sub-grid scale (SGS) fluctuations affecting the transport of mass, momentum and energy. In addition, chemical reactions mechanisms are strongly non-linear and

P. Domingo, A. Seltz, L. Vervisch

CORIA - CNRS, Normandie Université, INSA de Rouen, 76801 Saint-Etienne-du-Rouvray, France e-mail: domingo@coria.fr, vervisch@coria.fr, seltz@coria.fr. A. Seltz also with Safran Aircraft Engines, Site de Villaroche, Rond-Point René Ravaud-Réau, 77550 Moissy Cramayel, France

Z. Nikolaou

Computation-based Science and Technology Research Centre (CaSToRC), The Cyprus Institute, Nicosia, 2121, Cyprus. e-mail: z.nicolaou@ cyi.ac.cy 
specific tools must also be developed to compute burning rates over grids which are coarse at the scale of the thin reaction zones observed in flames [6, 7, 8, 9].

In large-eddy simulation (LES), a spatial filtering operation is applied to the first principle equations of aerothermochemistry and any quantity $\bar{\phi}(\underline{x}, t)$ resolved by the grid reads

$$
\bar{\phi}(\underline{x}, t)=\int_{-\infty}^{+\infty} \phi\left(\underline{x}^{\prime}, t\right) G_{\Delta}\left(\underline{x}^{\prime}-x\right) d x^{\prime},
$$

where $G_{\Delta}\left(\underline{x}^{\prime}-x\right)$ denotes a filtering operation of characteristic size $\Delta$ and $\phi(\underline{x}, t)$ is the fully resolved signal i.e., the solution that would be observed in a direct numerical simulation (DNS) resolving all the scales [10].

Because of unresolved SGS effects, the filtered value of any non-linear term $\overline{T(\phi)}$ differs from $T(\bar{\phi})$. So far, closures were developed in the literature in the form of mathematical expressions, providing estimation of the sub-grid scale effects from the knowledge of the quantities resolved by the grid, then $\overline{T(\phi)}=\mathcal{T}(\bar{\phi})$, where $\mathcal{T}(\bar{\phi})$ denotes the physical model used to express $\overline{T(\phi)}$ from the resolved field $\bar{\phi}$.

The application to turbulent flames of a different paradigm for modeling, which is based on a direct data analysis, is discussed in this chapter. Two main points are addressed:

- First, two methods of signal reconstruction based, respectively, on approximate inverse filtering and iterative deconvolution are discussed. In these two approaches, a tentative true signal $\phi^{\star}(\underline{x}, t)=\mathcal{L}_{\Delta}^{-1}[\bar{\phi}(\underline{x}, t)]$ is approximated from $\bar{\phi}(\underline{x}, t)$, the resolved field, to compute the non-linear terms which are then explicitly filtered,

$$
\overline{T(\phi)}(\underline{x}, t)=\overline{T\left(\mathcal{L}_{\Delta}^{-1}[\bar{\phi}(\underline{x}, t)]\right)} .
$$

- Second, a neural network is trained to relate the filtered non-linear terms (i.e., $\overline{T(\phi)})$ to three-dimensional distributions of resolved quantities surrounding the considered grid point $($ i.e., $T(\bar{\phi})$ or any function of $\bar{\phi})$. A mapping function $\mathcal{H}$ is then obtained so that

$$
\overline{T(\phi)}(\underline{x}, t)=\mathcal{H}\left[T\left(\bar{\phi}\left(\underline{x}_{1}, t\right)\right), \cdots, T\left(\bar{\phi}\left(\underline{x}_{N}, t\right)\right],\right.
$$

with $\underline{x}$ the $N$ points selected around $\underline{x}$ to build an input image for a neural network previously trained.

Both approaches are evaluated a priori from different DNS databases, for the prediction of unresolved SGS convective and diffusive fluxes and for the modeling of filtered chemical sources. 


\section{Flame filtering and signal reconstruction}

\subsection{Filtered scalar balance equation}

The budget equations for scalars describing flames (chemical species mass fractions, energy, temperature) may be cast in the generic form,

$$
\frac{\partial \rho \phi}{\partial t}+\nabla \cdot(\rho \mathbf{u} \phi)=\nabla \cdot(\rho D \phi)+\dot{\omega},
$$

where $\rho$ is the density $\mathbf{u}$ the velocity vector, $D$ a molecular diffusion coefficient and $\dot{\omega}$ a chemical source. Applying the filtering operation (1) leads to

$$
\frac{\partial \bar{\rho} \tilde{\phi}}{\partial t}+\nabla \cdot(\bar{\rho} \tilde{\mathbf{u}} \tilde{\phi})=\nabla \cdot(\bar{\rho} D(\tilde{\phi}) \nabla \tilde{\phi})+\overline{\dot{\omega}}+\nabla \cdot \tau,
$$

where $\tilde{\phi}=\overline{\rho \phi} / \bar{\rho}$ and

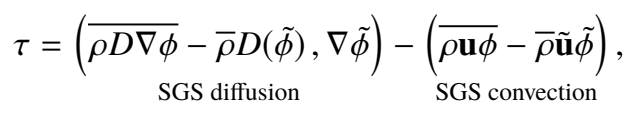

are two unresolved SGS fluxes. The molecular diffusion coefficient usually depends non-linearly on the flow thermochemical properties and the notation $D(\tilde{\phi})$ denotes $D$ computed from the resolved fields.

\subsection{Signal deconvolution}

Two methodologies for signal reconstructions are now discussed after introducing a relation useful to determine the filter size which should actually be applied according to the mesh resolution and the number of points required within the flame.

\subsubsection{Relation between flame thickness, filter size and mesh size}

For a mesh of characteristic size $h$ and a laminar premixed flame thickness $\delta_{L}$, the relation between the number of points within the filtered signal and the filter size $\Delta$ may be approximated as [11]:

$$
\frac{h}{\Delta}=\frac{1}{n} \sqrt{\frac{\pi}{6}+\frac{\delta_{L}^{2}}{\Delta^{2}}},
$$

where a Gaussian filtering operation has been assumed

$$
G(\underline{x})=\left(6 /\left(\pi \Delta^{2}\right)\right)^{3 / 2} \exp \left(-6 \underline{x} \cdot \underline{x} / \Delta^{2}\right),
$$




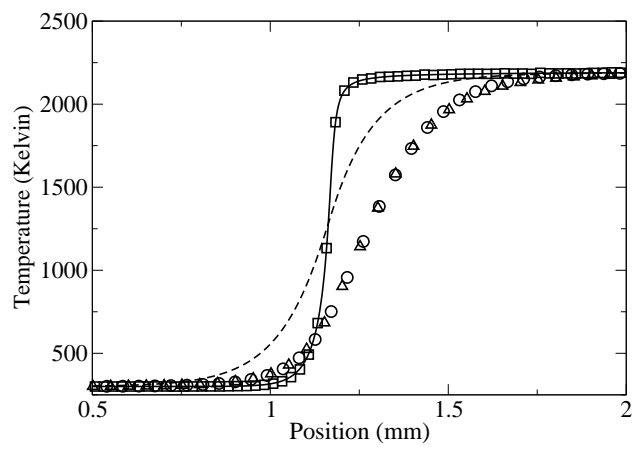

Fig. 1 Temperature profiles across a one-dimensional premixed flame. Line: $T(x)$; Square: $T^{\star}(x)$ from deconvolution (Eq. (10)); Dash: $\bar{T}(x)$; Circle: $\tilde{T}(x)$. Solving balance equation for filtered 1D flame from deconvolution Triangle: $\tilde{T}(x) . \Delta / \delta_{f l}=8$. Reprinted with permission [13].

with a flame front modeled with an error function. This relation shows that for a LES with $\Delta=5 \delta_{L}$, the mesh size should verify $h=0.75 \Delta / n$. Since $n>1, h$ is therefore expected smaller than the filter size applied to the scalar fields.

\subsubsection{Approximate deconvolution and explicite filtering (ADEF)}

Most filtering operations may be approximated as a diffusive process formulated implicitly

$$
\bar{\phi}(\underline{x}, t)=\phi(\underline{x}, t)+d \nabla^{2} \bar{\phi}(\underline{x}, t),
$$

with $d=\Delta^{2} / 24$ for a Gaussian filter [12]. From this relation, an approximate deconvolution operator is readily obtained in which all derivatives are resolved over the coarse mesh $(h<\Delta)$ :

$$
\phi^{\star}(\underline{x}, t)=\mathcal{L}_{\Delta}^{-1}[\bar{\phi}(\underline{x}, t)]=\bar{\phi}(\underline{x}, t)-d \nabla^{2} \bar{\phi}(\underline{x}, t) .
$$

To illustrate these filtering/deconvolution operations, Fig. 1 shows $T(x)$ (line), the temperature signal through a one-dimensional unstrained stoichiometric methane/air premixed flame, along with $\bar{T}(x)$ (dashed line) and $\tilde{T}(x)$ (circle). The deconvoluted signal $T^{\star}(x)$ (square) perfectly matches $T(x)$. Moreover, the solving of the budget equations of the one-dimensional filtered flame with all the non-linear terms computed from approximate deconvolution and filtering, returns the expected $\tilde{T}(x)$ (triangle). This was done here for $\Delta / \delta_{L}=8$ with a second order interpolation of the scalar signals inside computing cells $(h=0.5 \Delta)$ [13]. Flame deconvolution with an appropriate sub-grid interpolation or a regularisation procedure thus appears as a robust tool $[14,15]$. Sometimes deconvolution is also associated to a scale similarity hypothesis [16]. 


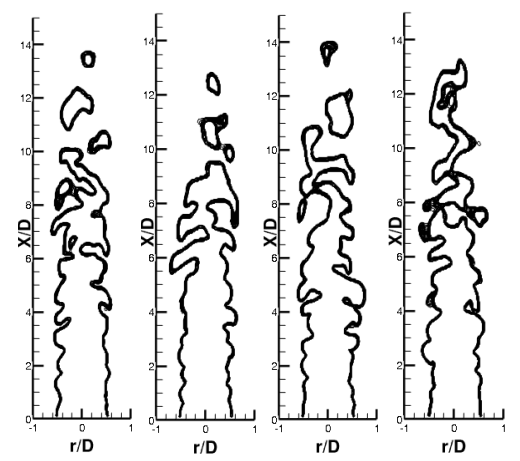

Fig. 2 Time sequence of iso-filtered source of progress variable, $\Delta t=1.8 \mathrm{~ms}$. Reprinted with permission [13].

Approximate deconvolution is easily generalised to three-dimensional turbulent flames. Figure 2 shows snapshots of the simulation of a stoichiometric methane/air turbulent bunsen flame studied experimentally [17]. These simulations are performed with the flow solver SiTCom [18], considering the Navier-Stokes equations in their fully compressible form together with a balance equation for $\tilde{c}$, a filtered progress variable ( $c=0$ in fresh gases and $c=1$ in burnt gases [6]). Tabulated detailed chemistry $[19,20]$ is coupled to deconvolution of this progress variable with Eq. (10) to simulate the premixed turbulent jet flame. The tabulation is based on the GRI3.0 [21] detailed chemistry, with a progress variable defined from $\mathrm{CO}, \mathrm{CO}_{2}, \mathrm{H}_{2} \mathrm{O}$ and $\mathrm{NO}_{\mathrm{x}}$ as in [22]. The thermochemical quantities are then read from a lookup table $\phi=\phi_{T}(c)$ and the filtered quantities may be written from deconvolution and explicit filtering,

$$
\bar{\rho} \tilde{\phi}=\overline{\rho^{\star} \phi_{T}\left(c^{\star}\right)}=\overline{\mathcal{L}_{\Delta}^{-1}[\bar{\rho}] \phi_{T}\left(\frac{\mathcal{L}_{\Delta}^{-1}[\bar{\rho} \tilde{c}]}{\mathcal{L}_{\Delta}^{-1}[\bar{\rho}]}\right)} .
$$

The convective terms are discretised with a fourth-order centered skew-symmetriclike scheme [23] and the diffusive terms with a fourth-order centered scheme. Time is advanced explicitly with a third order Runge-Kutta method and NSCBC boundary conditions [24] are imposed at inlet and outlet, with the measured profiles with synthetic turbulence [25] prescribed at inlet.

The time sequence $(\Delta t=1.8 \mathrm{~ms})$ of iso-burning rate (Fig. 2) illustrates the high level of resolved flame wrinkling in this jet flame simulation featuring $u^{\prime} / S_{L}=5$, with $u^{\prime}$ the characteristic velocity fluctuation and $S_{L}$ the laminar flame speed. The formation of pockets at the flame tip is also observed. The mesh resolution ${ }^{1}$ is of the order of $170 \mu \mathrm{m}$. The approximate deconvolution and explicit filtering approach coupled with tabulated chemistry allows for simulating the flame and predicting species mass fractions without resorting to any adjustment of model parameters.

${ }^{1}$ A resolution of $50 \mu \mathrm{m}$ would be necessary to fully resolve the flame (i.e., DNS) with tabulated chemistry and between $10 \mu \mathrm{m}$ and $80 \mu \mathrm{m}$ to resolve the Kolmogorov scale. 


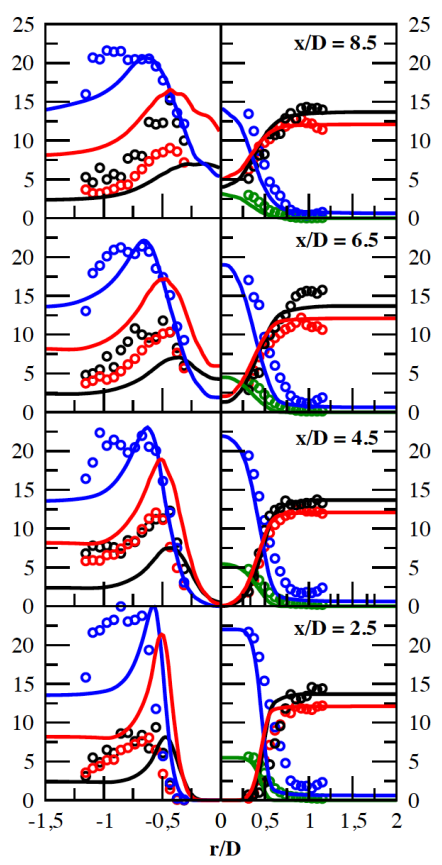

Fig. 3 Mass fractions in \%. Symbol measurements [17]. Line: LES. In each subfigure: Left: Red: $\mathrm{CO} \times 10$. Black: $\mathrm{H}_{2} \times 100$. Blue: $\mathrm{OH} \times 75$. Right: Blue: $\mathrm{O}_{2}$. Green: $\mathrm{CH}_{4}$. Red: $\mathrm{H}_{2} \mathrm{O}$. Black: $\mathrm{CO}_{2}$. Reprinted with permission [26].

Results are given in Fig. 3. The experimentalist reported a measurement error range between $8 \%$ and $15 \%$ for major species concentration and up to $25 \%$ for minor species [19,20]. The major species $\mathrm{O}_{2}, \mathrm{CH}_{4}, \mathrm{H}_{2} \mathrm{O}$ and $\mathrm{CO}_{2}$ agree with measurements within this range (Fig. 3-right). The chemistry tabulation limited to a progress variable as input does not include strain rate as a parameter in these simulations, explaining the higher departure for the minor species $\mathrm{CO}, \mathrm{H}_{2}$ and $\mathrm{OH}$.

\subsubsection{Iterative deconvolution and explicit filtering (IDEF)}

As an alternative to approximate deconvolution based on discretised inverse filtering, the Van Cittert algorithm is another well-established iterative deconvolution algorithm $[27,28]$. The calculation of derivatives is avoided by expressing the deconvoluted scalar field from

$$
\phi^{\star n+1}=\phi^{\star n}+b\left(\bar{\phi}-\overline{\phi^{\star n}}\right) .
$$

The initial condition of the iteration is $\phi^{\star 0}=\bar{\phi}$ and $b$ is defined so that the deconvoluted variable stays within specific bounds [11]. If $\phi_{l}$ and $\phi_{h}$ are the low and high bounds, on option consists in writting: 


$$
b=\left\{\begin{array}{l}
\frac{\phi^{\star n}-\phi_{l}}{\phi_{a}-\phi_{l}} \text { if } \phi \leq \phi_{a} \\
\frac{\phi^{\star n}-\phi_{h}}{\phi_{a}-\phi_{h}} \text { otherwise }
\end{array}\right.
$$

with the average value $\phi_{a}=\left(\phi_{l}+\phi_{h}\right) / 2$. The number of iterations is monitored through a volume averaged error $e_{v}=\left\langle\bar{\phi}-\overline{\phi^{\star}} \mid\right\rangle$, so that iterations can proceed as long as $e_{v}$ decreases, in practice for well resolved premixed flames $\left(\Delta<10 \delta_{L}\right)$, the iterations never exceed a few decades.

Correlations between conditionally averaged progress variable obtained from (12) compared to DNS values are shown in Fig. 4. The data are from a DNS of a methane/air planar premixed flame interacting with homogeneous turbulence [29]. In this DNS, $u^{\prime} / S_{L}=14$, the turbulent Reynolds number is $\operatorname{Re}_{T}=230$, the Damköhler number $\mathrm{Da}=1.17$, and the Karlovitz number $\mathrm{Ka}=13$.

These three-dimensional direct simulations have been performed using the SENGA2 code [30], which solves the compressible reacting flow Navier-Stokes equations using a 10th order finite difference scheme for interior points, and a 4th order Runge-Kutta scheme for the time- stepping. The DNS-filtered variables were sampled onto a LES $(h / \Delta=0.25)$ mesh using high-order Lagrange polynomials, values which are then used for the deconvolution [11]. The strong correlation between the prediction and the DNS reference (Fig. 4) confirms the potential of the iterative procedure for signal reconstruction. The time-averaged Pearson correlation coefficients are above 0.9850 for the three filter sizes $\left(\Delta=\delta_{L}, 2 \delta_{L}\right.$ and $\left.3 \delta_{L}\right)$ considered.

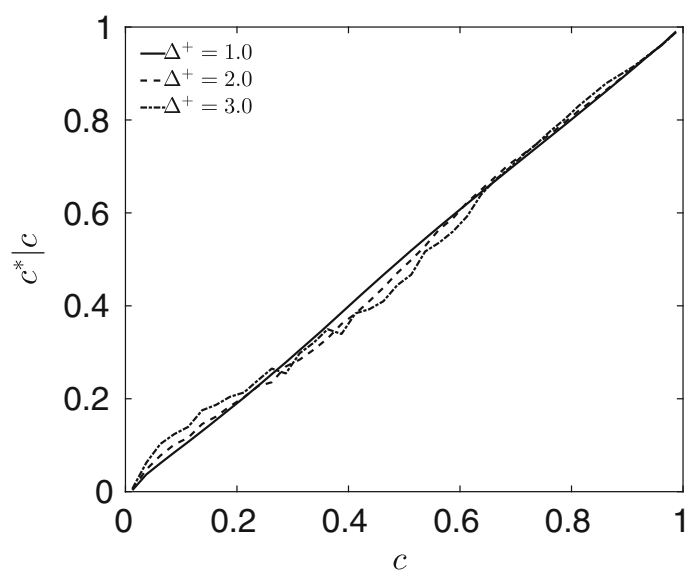

Fig. 4 Conditionally averaged progress variable as obtained from IDEF based on the actual value as obtained from the DNS. $\Delta^{+}=\Delta / \delta_{L}$ Reprinted with permission [11]. 
On this basis, the iterative deconvolution was further applied to compute unresolved SGS fluxes, as the convective one [31]

$$
\tau_{u}=\overline{\rho \mathbf{u} \phi}-\bar{\rho} \tilde{\mathbf{u}} \tilde{\phi}=\overline{(\rho \mathbf{u})^{\star} \frac{(\rho \phi)^{\star}}{\rho^{\star}}}-\bar{\rho} \tilde{\mathbf{u}} \tilde{\phi} .
$$

Evaluation against the same turbulent planar flame DNS configuration [29] of this SGS flux projected in the direction normal to the mean flame front is shown in Fig. 5. In this figure, comparisons against the well-established Clark model [32] are also added, this physical model reads

$$
f_{1 x}=\tau_{u} \cdot \mathbf{e}_{1}=\bar{\rho} \frac{\Delta^{2}}{12} \frac{\partial \tilde{u}_{1}}{\partial x_{j}} \frac{\partial \tilde{\phi}}{\partial x_{j}} .
$$

As expected the transition from counter gradient SGS turbulent transport to the usual gradient turbulent flux is found in the data by varying the turbulence intensity [33]. Indeed, $f_{1 x}>0$ (counter gradient transport) is seen in Fig. 5(a) for the progress variable larger than 0.3, and, $f_{1 x}<0$ (gradient transport) is observed in Fig. 5(b). The transport through the flame which was mainly driven by gas expansion for $u^{\prime} / S_{L}=3.18$ is rather governed by turbulent mixing for $u^{\prime} / S_{L}=9$. Interestingly, the deconvolution procedure perfectly reproduces this transition, while the physical model given by the relation (15) misses this very basic, but essential, property of SGS turbulent transport in premixed turbulent flame.

Following the same line, iterative deconvolution was also found effective to model the SGS stress tensor in premixed turbulent V-flame [34].

\section{Machine learning for turbulent combustion modeling}

\subsection{DNS embedded in LES database}

An additional DNS database was obtained in the configuration of the premixed turbulent bunsen flame discussed above in $\$ 2.2 .2$ [17]. This DNS is located downstream of the well-resolved LES of the first sections of the piloted premixed stoichiometric fuel-air round jet (Fig. 6), LES that provides turbulent flame conditions for the DNS inlet plane $[26,35,36]$. In this simulation, both LES and DNS are run simultaneously.

This dual simulation was achieved by embedding, inside the LES mesh, a zone where the resolution is sufficiently high to fully resolve the thin reaction zones and the smallest turbulent scales. The nozzle diameter is $\mathrm{D}=12 \mathrm{~mm}$, the jet Reynolds number is 24,000 (bulk nozzle velocity of $30 \mathrm{~m} \cdot \mathrm{s}^{-1}$ and turbulent kinetic energy of $3.82 \mathrm{~m}^{2} \cdot \mathrm{s}^{-2}$ ). The pilot is composed of fully burnt gases at $T_{b}=2200 \mathrm{~K}$. The LES mesh contains 171 million nodes for a domain $16 \mathrm{D} \times 8 \mathrm{D} \times 8 \mathrm{D}$, leading to a resolution of the order of $150 \mu \mathrm{m}$. The resolution in the DNS zone is uniformed at $50 \mu \mathrm{m}$, 

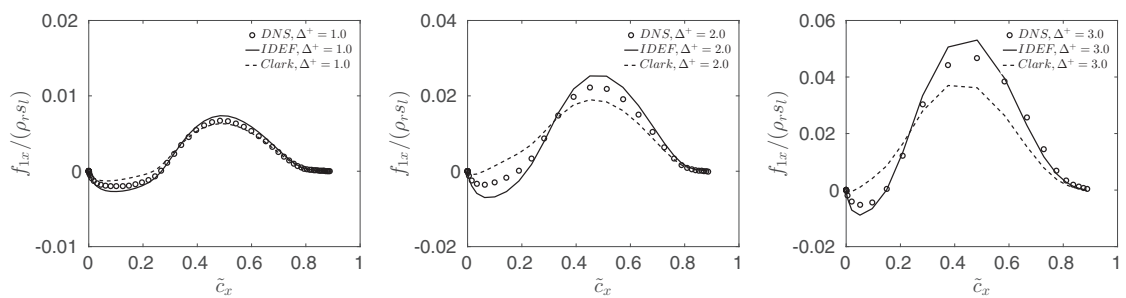

(a) $u^{\prime} / S_{L}=3.18, \operatorname{Re}_{T}=53, \mathrm{Da}=5.19, \mathrm{Ka}=1.39$.
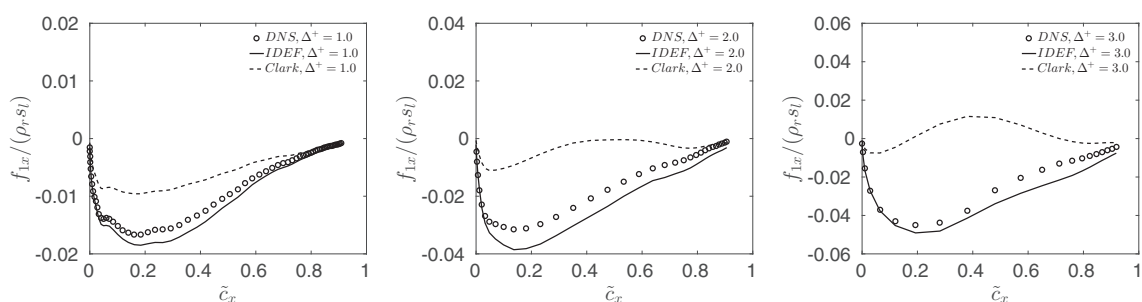

(b) $u^{\prime} / S_{L}=9, \operatorname{Re}_{T}=150, \mathrm{Da}=1.8, \mathrm{Ka}=6.62$.

Fig. 5 SGS convective flux in the direction normal to the mean flame front averaged in the transverse plane, plotted versus a progress variable ( $c_{x}=0$ in fresh gases and $c_{x}=1$ in fully burnt gases). ०: DNS. -: IDEF. --: Clark model [32]. Left: $\Delta / \delta_{L}=1$, Middle: 2, Right: 3. Reprinted with permission [31].

to secure a full resolution of the flow and flame scales for the chosen operating conditions [26].

Both LES and DNS rely on chemistry tabulation with a stoichiometric premixed flamelet with fresh gases at $T_{o}=300 \mathrm{~K}$ (GRI-3.0 mechanism [21] and progress variable defined as in [22]), without any SGS modeling in the DNS part. The flame thermal thickness based on the progress variable field is of the order of $\delta_{\mathrm{L}} \approx 400 \mu \mathrm{m}$. The mesh in the DNS zone is composed of 28.58 million nodes $(243 \times 343 \times 343)$, over a physical domain of $12 \mathrm{~mm} \times 18 \mathrm{~mm} \times 18 \mathrm{~mm}$. The DNS starts at $4.5 D$ downstream of the nozzle, and at $5.5 \mathrm{D}$ the mesh is coarsened again to progressively evolve toward LES resolution (Fig. 6). Modeling based on a progress variable presumed pdf approach is used for the chemical sources and species in the LES zones [37] and the SGS momentum fluxes are approximated with the Vreman model [38].

As for the jet flame LES of $\S 2.2 .2$, these simulations are performed using the flow solver SiTCom [18]. A Gaussian filtering operation (Eq. (8)), with filter size $\Delta=0.3 \mathrm{~mm}=0.75 \delta_{L}, \Delta=0.6 \mathrm{~mm}=1.50 \delta_{L}$ and $\Delta=0.9 \mathrm{~mm}=2.25 \delta_{L}$, is applied to the DNS variables in order to generate a priori LES filtered quantities. Thus the resolution of the a priori fields varies from well-resolved to coarse LES from the reaction zone point of view $(\Delta=0.9 \mathrm{~mm}$ is 18 times larger than the DNS grid resolution). 


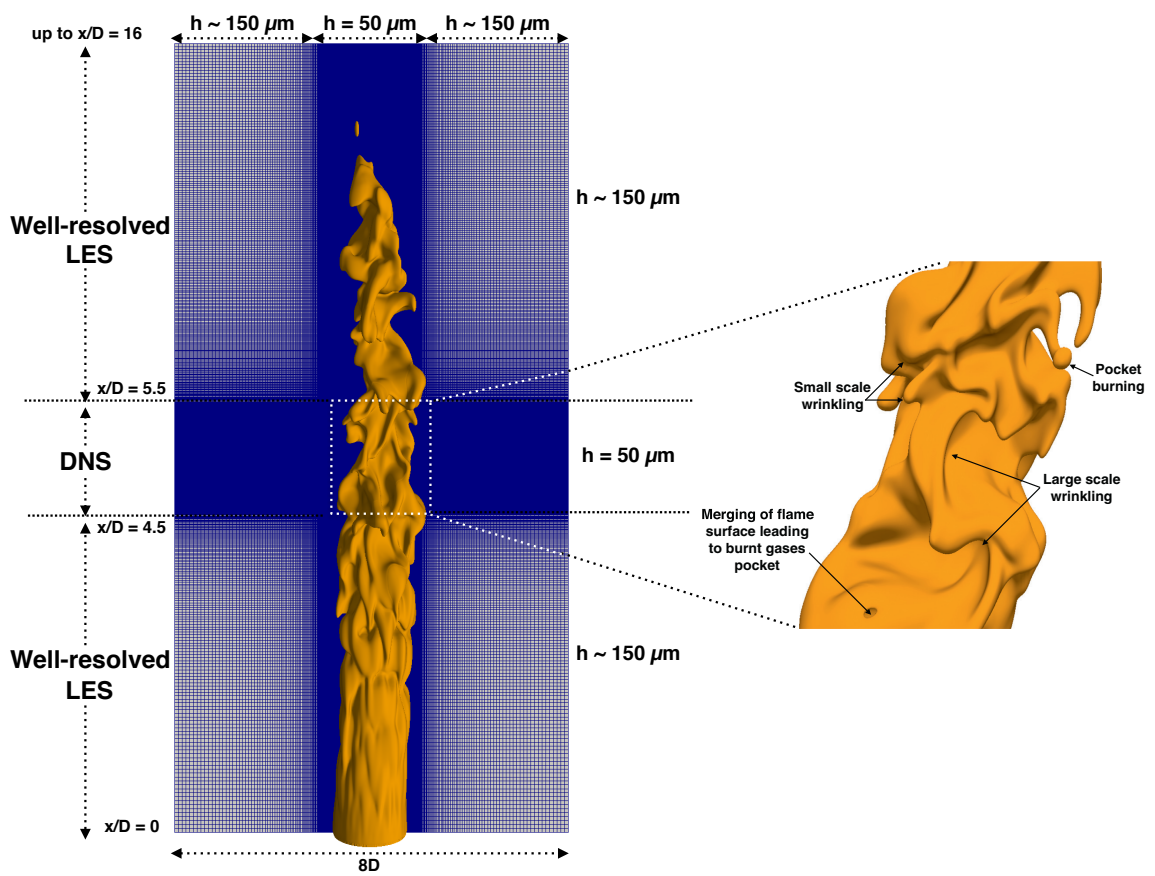

Fig. 6 LES-DNS snapshot of the jet-flame simulation [26]. Mesh and iso-progress variable $c=0.8$. $h$ : resolution. Right: zoom of iso- $c=0.8$ in the DNS zone (different angle view). Reprinted with permission [26].

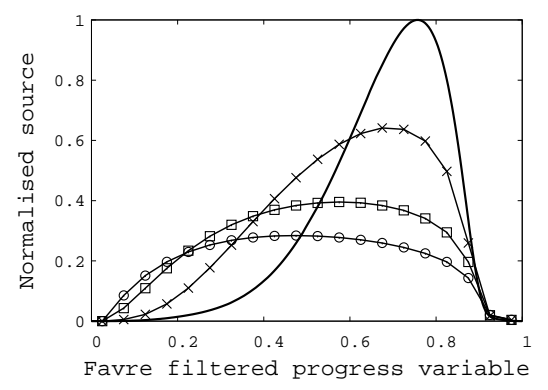

Fig. 7 Thick-line: $\dot{\omega}^{+}(\tilde{c})$ vs $\tilde{c}$ as obtained from a 1D laminar flame (tabulated chemistry). $\left\langle\overline{\dot{\omega}^{+}} \mid \tilde{c}\right\rangle$ from DNS vs $\tilde{c}$ for filter sizes $\times: 0.3 \mathrm{~mm}, \square: 0.6 \mathrm{~mm}, \circ: 0.9 \mathrm{~mm},\left(\delta_{L}=0.4 \mathrm{~mm}\right)$. Reprinted with permission [39].

In the DNS zone, the flame surface is highly convoluted (Fig. 6-right), with flame wrinkling covering a large spectrum of length scales, including complex flame topological properties such as pockets of fresh or burnt gases. 
Figure 7 shows $\left\langle\overline{\dot{\omega}^{+}} \mid \tilde{c}\right\rangle$, the statistical mean over the DNS domain of the normalised filtered progress variable source, conditioned on values of $\tilde{c}$. The subscript ${ }^{\prime+}$, denotes source terms normalised by their maximum value in the reference freelypropagating laminar premixed flame. The result obtained using a 1D laminar flame, $\dot{\omega}^{+}(\tilde{c})$, is also shown as a solid line. The maximum of $\left\langle\overline{\dot{\omega}^{+}} \mid \tilde{c}\right\rangle$ decreases while increasing the filter size and thus while increasing unresolved fluctuations [6]. Following the thickening of the filtered flame front, the response of this conditional filtered source term also spreads in progress variable space for increasing filter sizes, up to $\Delta=0.9 \mathrm{~mm}$. Figure 7 clearly demonstrate that $\overline{\dot{\omega}^{+}} \neq \dot{\omega}^{+}(\tilde{c})$ and not only the amplitude, but also the shape of the burning rate versus the progress variable, vary with the filter size. A simple re-scaling by a factor would therefore not be sufficient to properly model $\overline{\dot{\omega}}$ from $\dot{\omega}(\tilde{c})$. Similarly, Fig. 8 shows the conditional mean of the sum of the SGS convective and diffusive fluxes (Eq. (6)), which also requires modeling.

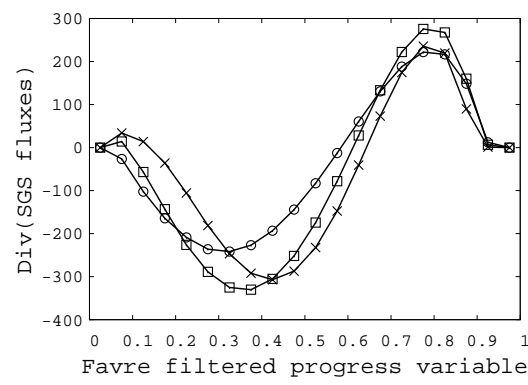

Fig. $8\langle\nabla \cdot \tau \mid \tilde{c}\rangle$ vs $\tilde{c}$. Filter size $\times: 0.3 \mathrm{~mm}, \square: 0.6 \mathrm{~mm}, \circ: 0.9 \mathrm{~mm}$. Reprinted with permission [39].

\subsection{Machine learning for turbulent combustion modeling}

The underlying idea discussed in this section consists of finding a mapping function $\mathcal{G}$, so that the filtered chemical source $\overline{\dot{\omega}}(\underline{x}, t)$ is constructed from the three dimensional distribution surrounding $\underline{x}$ of the chemical sources computed from the resolved field, i.e.,

$$
\overline{\dot{\omega}}(\underline{x}, t)=\mathcal{G}\left[\dot{\omega}\left(\tilde{c}\left(\underline{x}_{1}, t\right)\right), \cdots, \dot{\omega}\left(\tilde{c}\left(\underline{x}_{N}, t\right)\right)\right],
$$

where $N$ is the number of LES mesh cells involved in the mapping.

Similarly, a second mapping function $\mathcal{F}$ is sought to express $\nabla \cdot \tau$, the divergence of the SGS fluxes (Eq. 6), from the three-dimensional distribution of resolved diffusive budgets $\nabla \cdot\left(\bar{\rho} D_{c}(\tilde{c}) \nabla \tilde{c}\right)$ surrounding the $\underline{x}$ location, 


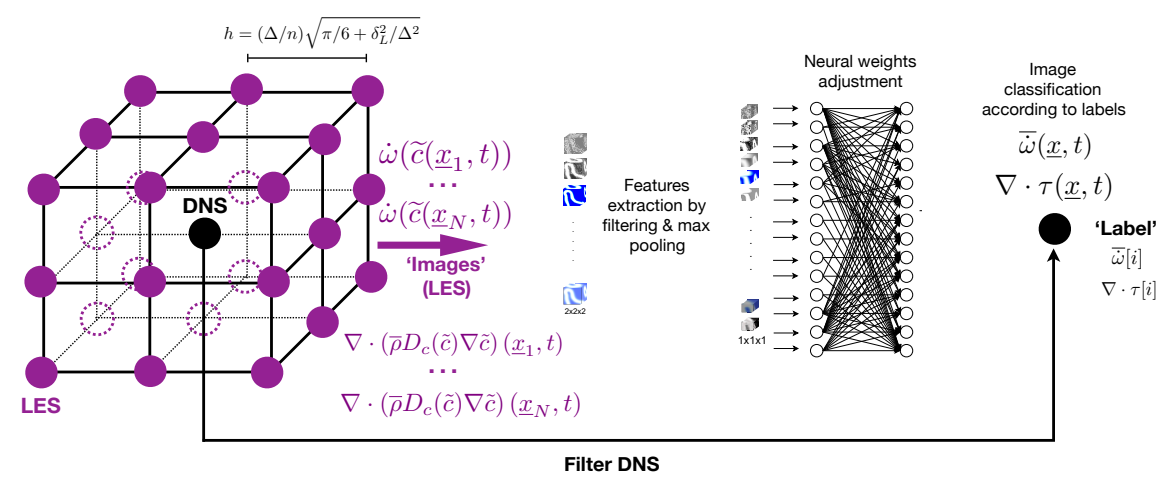

Fig. 9 CNN training from DNS, sketch of the database construction. Reprinted with permission [39].

$$
\nabla \cdot \tau(\underline{x}, t)=\mathcal{F}\left[\nabla \cdot\left(\bar{\rho} D_{c}(\tilde{c}) \nabla \tilde{c}\left(\underline{x}_{1}, t\right)\right), \cdots, \nabla \cdot\left(\bar{\rho} D_{c}(\tilde{c}) \nabla \tilde{c}\left(\underline{x}_{N}, t\right)\right)\right] .
$$

These two mapping function are built from convolutional neural networks (CNN), which conveniently allow for interpolating over a large number of reference values. Typically, if a set of $N_{L}$ reference values of filtered chemical sources $\overline{\dot{\omega}}[i]$ and divergence of the SGS fluxes $\nabla \cdot \tau[i]$ are known and associated through the mappings to well defined properties of the resolved field (here sources and diffusive budgets computed form the resolved scalar fields), well trained neural networks will return $\Omega_{i}(\underline{x}, t)$ and $T_{i}(\underline{x}, t)$ as the weights to be affected to the $i$-th values contributing to the filtered quantities, i.e.,

$$
\begin{aligned}
\overline{\dot{\omega}}(\underline{x}, t) & =\sum_{i=1}^{N_{L}} \Omega_{i}(\underline{x}, t) \times \overline{\bar{\omega}}[i], \\
\nabla \cdot \tau(\underline{x}, t) & =\sum_{i=1}^{N_{L}} T_{i}(\underline{x}, t) \times \nabla \cdot \tau[i] .
\end{aligned}
$$

The $\overline{\dot{\omega}}[i], \nabla \cdot \tau[i], \Omega_{i}$ and $T_{i}$ are calibrated by training the neural network from the DNS database. For a given filter size $\Delta$, a three-dimensional test-box of size $(2 h)^{3}$ is built around every of the $\mathrm{M}=28.58$ million DNS nodes, where $h$ is defined from Eq. (7). Figure 9 illustrates this process. The test box is centered at $\underline{x}$ and contains $N=27$ points which hold the three-dimensional distributions of $\overline{\dot{\omega}}\left(\tilde{c}\left(\underline{x}_{j}, t\right)\right)$ and $\nabla \cdot\left(\bar{\rho} D_{c}(\tilde{c}) \nabla \tilde{c}\right)\left(\underline{x}_{j}, t\right)$, for $j=1, \cdots, N$. These data are stored and constitute the 'images' that will be processed by the CNN. From the DNS, the 'labels' of each $i$-th image are $\overline{\dot{\omega}}[i]=\overline{\dot{\omega}}(\underline{x}, t)$ and $\nabla \cdot \tau[i]=\nabla \cdot \tau(\underline{x}, t)$ for $i=1, \cdots, N_{L}$. In the present work $N_{L}=2000$.

Two networks of similar structures (same number of layers, convolution kernels, etc.) are trained, one for the chemical source and one for the SGS fluxes. For each value of $\Delta$, the following procedure is applied: 


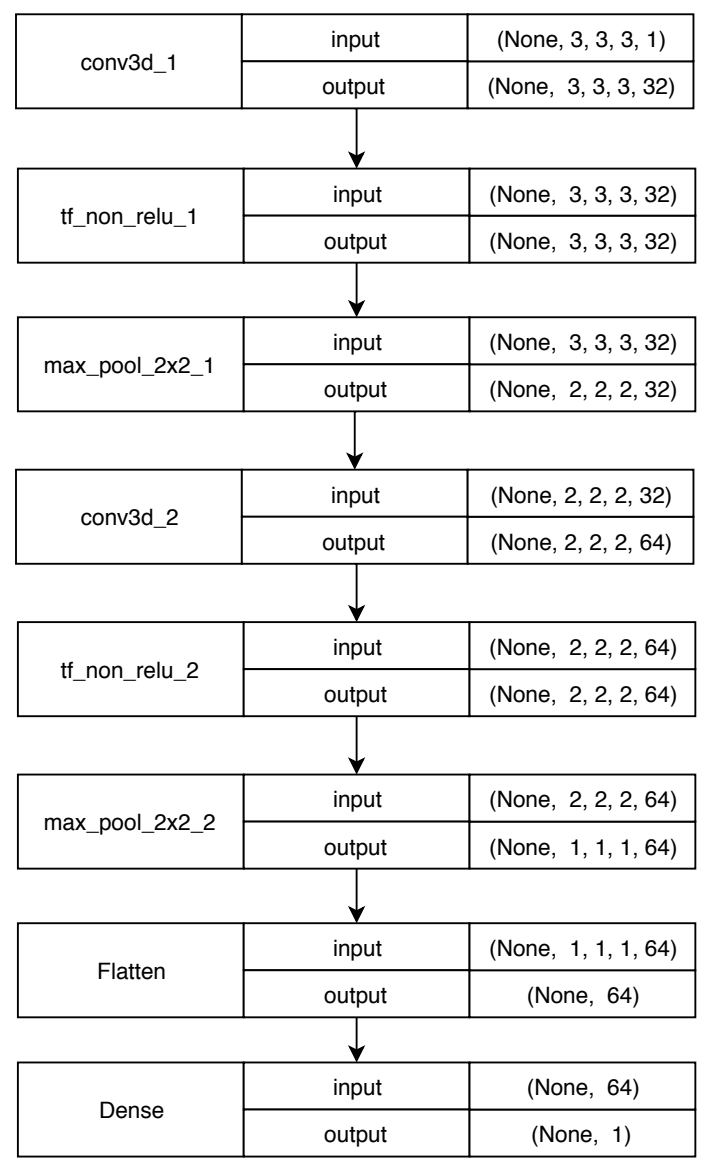

Fig. 10 Typical structure of a convolutional network for scalar deconvolution. More details on the TensorFlow functions used may be found in [39]. Reprinted with permission [39].

- First, 1000 images with their associated $\overline{\dot{\omega}}[i]$ and $\nabla \cdot \tau[i]$ values are drawn. 20 values of $c^{*}$ uniformly distributed between 0 and $1\left(\Delta c^{*}=0.05\right)$ are defined. For each value of $c^{*}, 50$ images are selected randomly such that $\tilde{c}(\underline{x}, t) \in\left[c^{*}-\right.$ $\left.\Delta c^{*} / 2 ; c^{*}+\Delta c^{*} / 2\right]$ ( $\underline{x}$ is at the center of the test box, see Fig. 9).

- To avoid overfitting, uncorrelated random perturbations are added to the images, $\dot{\omega}\left(\tilde{c}\left(\underline{x}_{j}, t\right)\right)$ and $\nabla \cdot\left(\bar{\rho} D_{c}(\tilde{c}) \nabla \tilde{c}\right)\left(\underline{x}_{j}, t\right)$ for $j=1, \cdots, N=27$, as $10 \%$ of their maximum in the test box, thus a second image is available for each label. $N_{L}=$ 2000 images then exist for 1000 labels.

Using two filter sizes, the database to train the network contains 4000 images and 2000 labels and for every quantity learned, a set of $27 \times 4000=108000$ data $\left(\dot{\omega}\left(\tilde{c}\left(\underline{x}_{j}, t\right)\right)\right.$ and $\left.\nabla \cdot\left(\bar{\rho} D_{c}(\tilde{c}) \nabla \tilde{c}\right)\left(\underline{x}_{j}, t\right)\right)$ is involved, data which are associated to the 2000 reference labels $\left(\overline{\dot{\omega}}[i]\right.$ and $\nabla \cdot \tau[i]$ for $\left.i=1, \cdots, N_{L}\right)$. 


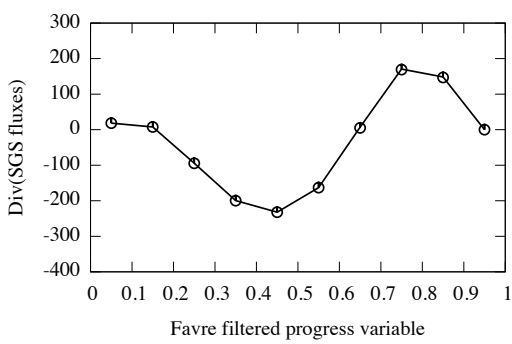

(a) $\Delta=0.30 \mathrm{~mm}$ (trained)

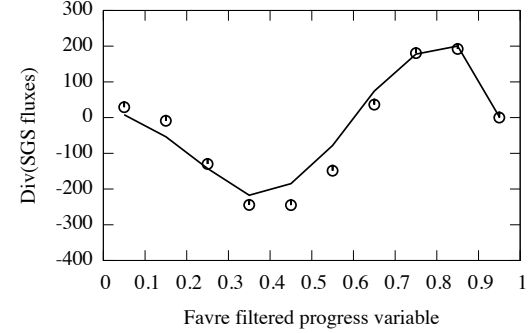

(b) $\Delta=0.45 \mathrm{~mm}$ (untrained)

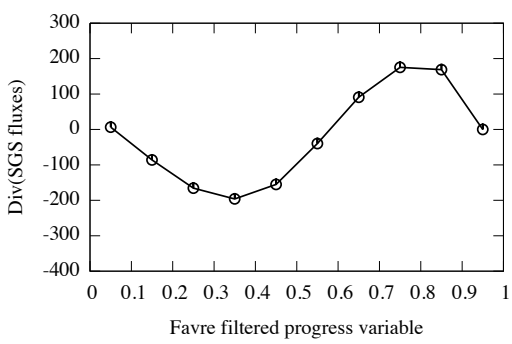

(c) $\Delta=0.90 \mathrm{~mm}$ (trained)

Fig. $11\langle\nabla \cdot \tau \mid \tilde{c}\rangle$ vs $\tilde{c}$. Symbols: DNS reference. Line: CNN prediction. Reprinted with permission [40].

During the training phase, a series of convolution/sampling operations are performed iteratively, in which the neural weights are adjusted until a satisfying minimal error is found between the value of the labels and the neural network prediction. A series of convolution/sampling operations are applied to the database, to extract its features using a number of different kernels [41, 42]:

- Each image is first convoluted with 32 different filter kernels obtained with random values from a truncated normal distribution. Meaningful values of the returned features are extracted with a max pooling non-linear function to avoid excessive computational costs.

- The process is repeated with 64 filters, decomposing the image into several most meaningful features, needed for seeking out the inner properties of the fluxes and sources.

- Two fully connected layers are built to process the 64 obtained features, and to classify the image by giving the probability it belongs to each label.

The training of the network was conducted using the TensorFlow (www.tensorflow.org) library and breakdown of the network structure is given in Fig. 10. The training error function is based on cross entropy [43] and this training is controlled by the Adam optimizer [44] for stochastic gradient descent, with a learning rate of $10^{-4}$.

In using the networks, the $N=27$ values of the chemical sources and of the divergence of the fluxes computed from the resolved progress variable field in the 


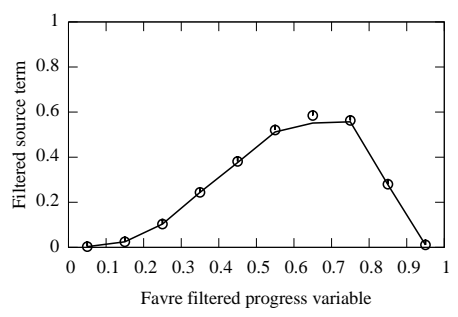

(a) Trained database. $\Delta=0.30 \mathrm{~mm}$

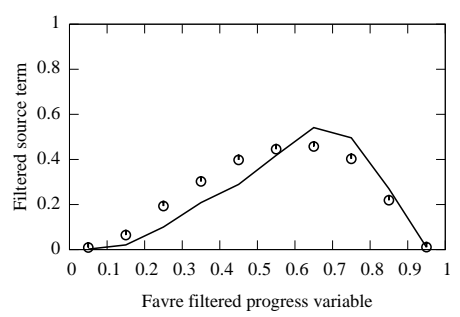

(c) Untrained database. $\Delta=0.45 \mathrm{~mm}$

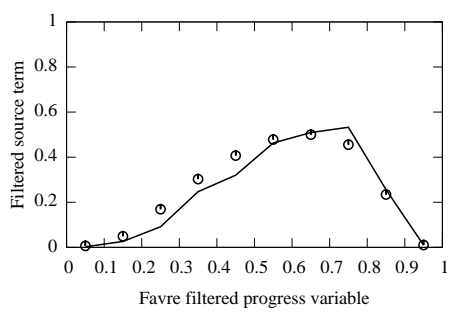

(b) Untrained database. $\Delta=0.40 \mathrm{~mm}$

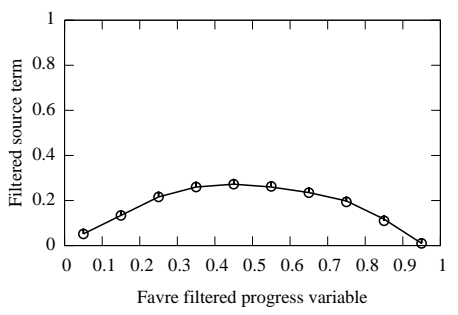

(d) Trained database. $\Delta=0.90 \mathrm{~mm}$

Fig. $12\left\langle\overline{\dot{\omega}^{+}} \mid \tilde{c}\right\rangle$ vs $\tilde{c}$. Symbols: DNS reference. Line: CNN prediction.

test box surrounding the LES cell (Fig. 9), are the input. For each filter size, 1000 filtered DNS fields are used for a priori tests (the noised images of the training phase do not enter these tests).

Figure 11 shows the comparison between the conditional means of the predicted divergence of the fluxes and their DNS counterpart. For the trained filter size, the CNN returns the expected value, for the untrained one some departure is observed, but compared to what can be observed in some case using a physical model, results are more than encouraging. (Compare for instance these results against those of Fig. 5 with the Clark model.)

The filtered chemical source are also very well predicted both the shape and the amplitudes are recovered by the CNN (Fig. 12). Interestingly, even the data corresponding to the filter sizes that were not used for training $(0.4 \mathrm{~mm}$ and 0.45 $\mathrm{mm}$ ) are quite well reproduced.

\section{Conclusion}

Large datasets from direct numerical simulation have been mostly used so far to explore reacting flow physics and to test well-established or novel modeling ideas. With rapidly growing computing facilities, followed by the development of well- 
resolved large eddy simulations for real combustion systems, the possibility of relying on modeling based on a direct analysis of the signals is now emerging.

Instead of expressing unclosed terms from algebraic relations involving quantities resolved by the mesh, deconvolution may be used to directly reconstruct the nonlinear terms to close the scalar balance equations, then some form of sub-grid scale interpolation is required. Both discretised and iterative deconvolutions have been found valuable along these lines.

In addition, the capabilities of machine learning tools to estimate unclosed terms from the resolved ones have been demonstrated, which opens many perspectives such as the coupling between advanced computational flame dynamics tools and artificial intelligence.

The deconvolution of scalar signals and the direct mapping form LES resolved scales to unknown filtered terms from CNN do not operate at the same level (signal reconstruction or direct prediction) and could thus be combined as complementary tools.

\section{References}

1. R. W. Bilger, S. B. Pope, K. N. C. Bray, and J. F. Driscoll. Paradigms in turbulent combustion research. Proc. Combust. Inst, 30(1):21-42, 2005.

2. L. Y. M. Gicquel, G. Staffelbach, and T. Poinsot. Large Eddy Simulations of gaseous flames in gas turbine combustion chambers. Progress in Energy and Combustion Science, 38(6):782817, 2012.

3. T. Poinsot. Prediction and control of combustion instabilities in real engines. Proc. Combust. Inst., 36(1):1-28, 2017.

4. E. Mastorakos. Forced ignition of turbulent spray flames. Proc. Combust. Inst., 36(2):23672383, 2017.

5. C. Locci, L. Vervisch, B. Farcy, and N. Perret. Selective non-catalytic reduction (SNCR) of nitrogen oxide emissions: A perspective from numerical modeling. Flow Turbulence and Combust., 100(2):301-340, 2018.

6. K. N. C. Bray. The challenge of turbulent combustion. Symp. (Int.) on Combust., 26:1-26, 1996.

7. N. Peters. Turbulent Combustion. Cambridge University Press, 2000.

8. D. Veynante and L. Vervisch. Turbulent combustion modeling. Prog Energy Combust Sci, 28:193-266, 2002

9. H. Pitsch. Large Eddy Simulation of turbulent combustion. Annual Review of Fluid Mechanics, 38:453-482, 2006.

10. M. Lesieur, O. Métais, and P. Comte. Large-Eddy Simulations of Turbulence. Cambridge University Press, Cambridge UK, 2005.

11. Z. Nikolaou and L. Vervisch. A priori assessment of an iterative deconvolution method for les sub-grid scale variance modelling. Flow Turbulence and Combust., 101(1):33-53, 2018.

12. F. Katopodes, R. L. Street, M. Xue, and J. H. Ferziger. Explicit filtering and reconstruction turbulence modeling for large-eddy simulation of neutral boundary layer flow. $J$. of the Atmospheric Sciences, 62(7):2058-2077, 2004.

13. P. Domingo and L. Vervisch. Large Eddy Simulation of premixed turbulent combustion using approximate deconvolution and explicit flame filtering. Proc. Combust. Inst., 35(2):1349-1357, 2015.

14. Q. Wang and M. Ihme. Regularized deconvolution method for turbulent combustion modeling. Combust. Flame, 176:125-142, 2017. 
15. C. Mehl, J. Idier, and B. Fiorina. Evaluation of deconvolution modelling applied to numerical combustion. Combust. Theory Modelling, 22(1):38-70, 2018.

16. A. W. Vreman, R. J. M. Bastiaans, and B. J. Geurts. A similarity sub-grid model for premixed turbulent combustion. Flow Turbulence Combust., 82(2):233-248, 2009.

17. Y.-C. Chen, N. Peters, G. A. Schneemann, N. Wruck, U. Renz, and M. S. Mansour. The detailed flame structure of highly stretched turbulent premixed methane-air flames. Combust. Flame, 107(3):223-244, 1996.

18. L. Bouheraoua, P. Domingo, and G. Ribert. Large-eddy simulation of a supersonic lifted jet flame: Analysis of the turbulent flame base. Combust. Flame, 179:199-218, 2017.

19. O. Gicquel, N. Darabiha, and D. Thevenin. Laminar premixed hydrogen / air counterflow flame simulations using flame prolongation of ILDM with differential diffusion. Proc. Comb. Inst., 28:1901-1908, 2000.

20. J. A. van Oijen, F. A. Lammers, and L. P. H. de Goey. Modeling of complex premixed burner systems by using flamelet-generated manifolds. Combust. Flame, 127(3):2124-2134, 2001.

21. G. P. Smith, D. M. Golden, M. Frenklach, N. W. Moriarty, B. Eiteneer, M. Goldenberg, C. T. Bowman, R. K. Hanson, S. Song, W. C. Gardiner, V. V. Lissianski, and Z. Qin. Technical report, 1999. http://www.me.berkeley.edu/gri-mech/.

22. G. Godel, P. Domingo, and L. Vervisch. Tabulation of nox chemistry for large-eddy simulation of non-premixed turbulent flames. Proc. Combust. Inst., 32:1555-1561, 2009.

23. F. Ducros, F. Laporte, T. Soulères, V. Guinot, P Moinat, and B. Caruelle. High-order fluxes for conservative skew-symmetric-like schemes in structured meshes: application to compressible flows. J. Comput. Phys., 161:114-139, 2000.

24. G. Lodato, P. Domingo, and L. Vervisch. Three-dimensional boundary conditions for direct and large-eddy simulation of compressible viscous flows. J. Comput. Phys, 227(10):5105-5143, 2008.

25. M. Klein, A. Sadiki, and J. Janicka. A digital filter based generation of inflow data for spatially developing direct numerical or large eddy simulations. J. Comp. Physics, 186(2):652-665, 2002.

26. P. Domingo and L. Vervisch. Dns and approximate deconvolution as a tool to analyse onedimensional filtered flame sub-grid scale modeling. Combust. Flame, 177:109-122, 2017.

27. P. H. Van Cittert. Zum einfluss der spaltbreite auf die intensitätverteilung in spektralinien. II, Z. Physik, 69:298-308, 1931.

28. P. A. Jansson. In Deconvolution with applications in spectroscopy, pages 67-134. Academic ch., New York, 1984.

29. Z. M. Nikolaou and N. Swaminathan. Direct numerical simulation of complex fuel combustion with detailed chemistry: physical insight and mean reaction rate modeling. Combust. Sci. Tech., 187:1759-1789, 2015.

30. R. S. Cant. Senga2 user guide. cued/a?thermo/tr67. Technical report, 2012.

31. Z. Nikolaou, R. S. Cant, and L. Vervisch. Scalar flux modelling in turbulent flames using iterative deconvolution. Phys. Rev. Fluids., 3(4):043201, 2018.

32. R. A. Clark. Evaluation of sub-grid scalar models using an accurately simulated turbulent flow. J. Fluid Mech., 91(1), 1979.

33. D. Veynante, A. Trouvé, K.N.C. Bray, and T. Mantel. Gradient and counter-gradient scalar transport in turbulent premixed flames. J. Fluid Mech., 332:263-293, 1997.

34. Z. M. Nikolaou, Y. Minamoto, and L. Vervisch. Unresolved stress tensor modeling in turbulent premixed v-flames using iterative deconvolution: An a priori assessment. Phys. Rev. Fluids, 4:063202, 2019.

35. L. Cifuentes, C. Dopazo, J. Martin, P. Domingo, and L. Vervisch. Local volumetric dilatation rate and scalar geometries in a premixed methane-air turbulent jet flame. Proc. Combust. Inst., 35(2):1295-1303, 2015.

36. L. Cifuentes, C. Dopazo, J. Martin, P. Domingo, and L. Vervisch. Effects of the local flow topologies upon the structure of a premixed methane-air turbulent jet flame. Flow Turbulence Combust., 96(2):535-546, 2016.

37. P. Domingo, L. Vervisch, and D. Veynante. Large-Eddy Simulation of a lifted methane-air jet flame in a vitiated coflow. Combust. Flame, 152(3):415-432, 2008. 
38. A. W. Vreman. An eddy-viscosity subgrid-scale model for turbulent shear flow: Algebraic theory and applications. Phys. Fluids., 16(10):3670-3681, 2004.

39. A. Seltz, P. Domingo, L. Vervisch, and Z. Nikolaou. Direct mapping from LES resolved scales to filtered-flame generated manifolds using convolutional neural networks. Combust. Flame, 210:71-82, 2019.

40. Z. Nikolaou, C. Chrysostomou, L. Vervisch, and R. S. Cant. Progress variable variance and filtered rate modelling using convolutional neural networks and flamelet methods. Flow Turbulence Combust. https://doi.org/10.1007/s10494-019-00028-w, 2019.

41. J. Schmidhuber. Deep learning in neural networks: An overview. Neural Networks, 61:85-117, 2015.

42. Y. Lecun, Y. Bengio, and G. Hinton. Deep learning. Nature, 521:436-444, 2015.

43. P.-T. de Boer, D. P. Kroese, S. S. Mannor, and R. Y. Rubinstein. A tutorial on the cross-entropy method. Annals of operations research, 134(1):19-67, 2005.

44. D. P. Kingma and J. L. Ba. ADAM: A method for stochastic optimization. https://arxiv.org/pdf/1412.6980, 2017. 